\title{
EVALUACIÓN DE LOS RIESGOS DE AFECTACIÓN POR EL ESTABLECIMIENTO DE GRANJAS ATUNERAS EN RELACIÓN CON LA DISTRIBUCIÓN ESPACIAL DE CETÁCEOS EN EL GOLFO DULCE, COSTA RICA
}

\author{
Lenin E. Oviedo Correa ${ }^{1,2,4 *}$, Juan Diego Pacheco ${ }^{3,4}$ y David Herra Miranda ${ }^{4}$
}

\begin{abstract}
RESUMEN
Este estudio trata sobre la distribución espacial de depredadores marinos topes, particularmente cetáceos odontocetos y misticetos migratorios. Se describe el riesgo potencial de afectación por el establecimiento de instalaciones asociadas con operaciones locales de maricultura: jaulas de engorde para atún. Por medio de un enfoque teórico y empírico se establece cuantitativamente como la profundidad máxima, un factor limitante en la distribución y definición del nicho ecológico de cetáceos, se solaparía en valores con el área propuesta para el establecimiento de las jaulas, lo que induciría dos escenarios probables de afectación: el desplazamiento de las poblaciones de depredadores de un hábitat-nicho de alimentación potencial y la interacción progresiva entre los depredadores con la fauna acompañante alrededor de las instalaciones de la operación de cultivo, lo anterior implicaría un riesgo perenne de captura incidental de dichos depredadores. Adicionalmente, la presencia de ballenas jorobadas durante la temporada de lluvia en zonas aledañas a Punta Banco, se asociaría a una ruta migratoria desde las zonas de alimentación en Chile-Antártica, que pasaría por el área proyectada para el establecimiento de la operación de maricultura. Los resultados ilustran la necesidad de seguir un principio precautorio y promover la reubicación de proyecto de maricultura en áreas de menor valor en términos de biodiversidad marina.
\end{abstract}

Palabras claves: Distribución, maricultura, cetáceos odontocetos, ballena jorobada, nivel trófico.

\begin{abstract}
This contribution deals with the spatial distribution of marine top predators, particularly odontocete and migratory misticete cetaceans. The study describes the potential risk of disturbances due to the establishment of mariculture operations and corresponding infrastructure: tuna feed lots. Through a mixed theoretical and empirical approach, this research corroborates how the maximum depth, a limiting factor in the definition of cetacean trophic niches off the study area, overlaps with the values for depths from an area proposed as the focal location of a tuna feed lot facility. The latter implies two particular scenarios: the translocation of the local predator population from a potential foraging habitat-niche, and the progressive negative interaction between predators and the accompanying fish fauna around of the cages, with a high probability of incidental capture of those predators by entanglement. Additionally, the occurrence of humpback whales during the rainy season associated with a migratory route from feeding grounds off Chile-Antarctica, that would pass through the area projected to harbor the mariculture operation. The results illustrate the need of considering a precautionary approach and relocate mariculture projects to areas of lesser value in terms of marine biodiversity.
\end{abstract}

Keywords: Distribution, mariculture, cetaceans odontocetes, humpback whales, trophic level.

1 PROCMAR, Programa de Maestría de Ciencias Marinas y Costeras, Universidad Nacional (UNA), Costa Rica.

2 Fundación Vida Marina, Drake Bay, Costa Rica.

3 Escuela de Biología, Universidad Nacional (UNA).

4 Vida Silvestre Golfo Dulce, El Chontal, Rincón de Osa, Golfo Dulce, Costa Rica. golfodulcewildlife@ gmail.com*

Recibido 29-V-2009

Aceptado 30-IX-2009

DOI: http://dx.doi.org/10.15359/revmar.1.9

Rev. Mar. y Cost. ISSN 1659-455X. Vol. 1. 159-174, Diciembre 2009. 


\section{INTRODUCCIÓN}

Las aguas alrededor de la Península de Osa, por sus características ecogeográficas promueven la presencia y el establecimiento de poblaciones de cetáceos. La riqueza de especies abarca aproximadamente 13 especies (May-Collado et al. 2005; Fernández et al. 2007), algunas de carácter residente (delfín manchado pantropical-Stenella attenuata, delfin nariz de botella-Tursiops truncatus) y muchas de hábitos transicionales (ballena jorobada-Megaptera novaeangliae). Las poblaciones residentes a su vez pueden tener una presencia constante o asociada a un patrón de estacionalidad, como son los casos de delfines comunes asociados a temporadas de vientos activos que promueven afloramientos y niveles significativos de productividad (Fernández et al. 2007; Fernández \& Oviedo, 2009).

La posibilidad de hábitats diferenciales en términos físicos se traduce a su vez en nichos ecológicos diversos, con la presencia de depredadores topes, como los cetáceos, que a su vez se asocian a cadenas tróficas de complejidad variada por cada hábitat potencial (Evans, 2002; Fiedler, 2002; Forcada, 2002; Begon et al. 2006). El uso de unidades de paisajes para la evaluación, seguimiento y conservación de la diversidad de las especies, permite un análisis integral de ecosistemas complejos, ya que en las unidades de paisajes (hábitats diferenciales), las actividades humanas y sus consecuencias (contaminación, degradación, cambios y fragmentación de ecosistemas) tienen efectos perceptibles y cuantificables que usualmente eluden análisis de carácter muy local (Ramesh, 2008).

El incremento de la acuicultura en áreas costeras debería estar enmarcado por un mayor conocimiento de los posibles efectos y consecuencias en el medio ambiente, donde se desarrolla la operación. La acuicultura es una actividad que puede provocar cambios estructurales en las comunidades bióticas del hábitat que sustenta la operación, principalmente causando un enriquecimiento en materia orgánica del medio, y a su vez, una atracción de numerosas especies de peces y con ello a sus depredadores (Díaz-López et al. 2008). Incluso, las actividades de acuicultura en tierra firme (en específico aquellas que particularmente no consideran los aspectos ambientales claves de riesgo dentro de la operación) tienen consecuencias para los ecosistemas marinos relacionados con el incremento de nutrientes y la polución.

Estudios centrados en el impacto de la acuicultura en la fauna marina (y en particular cetáceos) han hecho énfasis en las consecuencias derivadas tanto por desplazamiento de hábitat de uso potencial, como por la interacción de depredadores con las instalaciones de la operación en desarrollo (Kemper et al. 2003). Lo anterior está especialmente condicionado a la modalidad de la operación o proyecto de acuicultura, en particular, la maricul- 
tura con jaulas flotantes es una de las actividades que como consecuencia generan dos tipos de interacción negativa: aquellas dirigidas al daño de instalaciones por depredación del stock en cultivo, y las relacionadas con depredación de fauna acompañante (un efecto colateral por enriquecimiento de la columna de agua). En ambas instancias, el riesgo más crítico es el de la captura incidental del depredador, al enredarse con las estructuras de contingencia (Kemper \& Gibbs, 2001; Kemper et al. 2003).

Este estudio hace énfasis en determinar las características físicas de los nichos ecológicos asociados con cetáceos, primeramente en términos de su agrupación y categorización por nivel trófico, utilizando como caso de estudio el proyecto de maricultura propuesto para la localidad de Punta Banco en la costa del Pacífico Sur de Costa Rica. La finalidad de esta contribución es proveer evidencias cuantitativas sobre el riesgo potencial de interacción negativa y desplazamiento de hábitat de mamíferos marinos en la zona propuesta para el establecimiento de las jaulas de engorde.

\section{MATERIALES Y MÉTODOS}

El análisis que se presenta en esta contribución es un enfoque mixto de bases teóricas en adición a observaciones empíricas. El estudio combina elementos inherentes a la ecología trófica y espacial de cetáceos en el área de estudio (Pacífico Sur, Costa Rica,

\section{Figura 1}

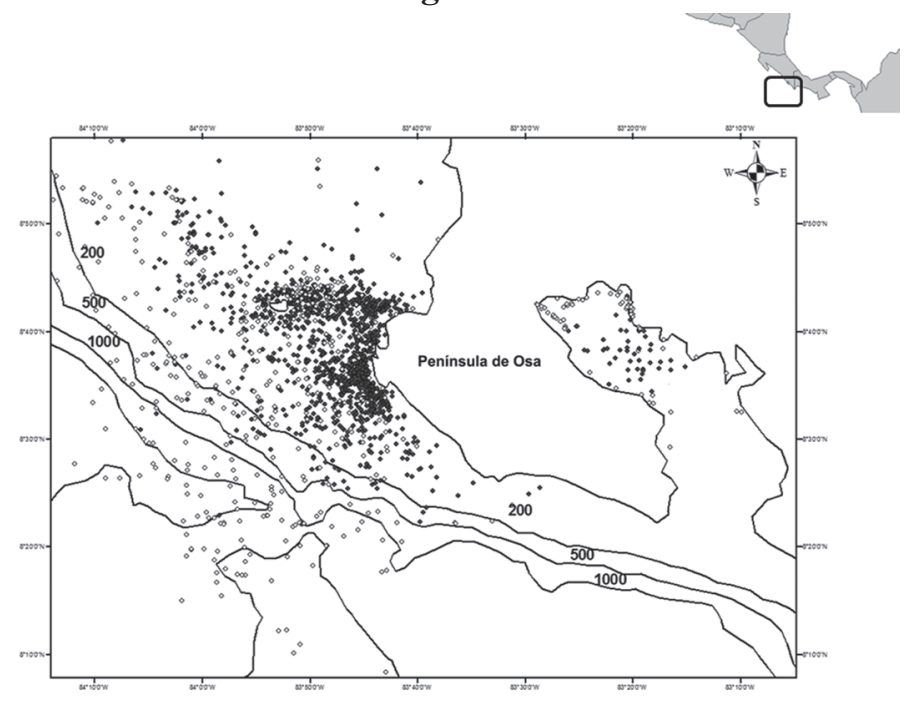

Área de estudio en el Pacífico sur de Costa Rica. Los puntos negros representan avistamientos de delfín manchado, los claros representan otras especies de odontocetos.

Study area in the southern Pacific coast of Costa Rica. Black dots are spotted dolphin sightings; light ones, other odontocete species. 
incluyendo el Golfo Dulce y las zonas aledañas a Punta Banco, Figura 1). Lo anterior se basa principalmente en lo propuesto por Oviedo (2008) y Oviedo et al. (in rev.) sustentado por los niveles tróficos descritos en Pauly et al. (1998). Para detalles con respecto a dietas y otros elementos metodológicos se sugiere revisar los trabajos anteriormente mencionados. Los análisis se ejecutaron utilizando seis años de observaciones en los ámbitos marinos y costeros en áreas de la Península de Osa y sus adyacencias (base de datos Fundación Vida Marina 2001-2006), complementados con cuatro años de registros (2005-2009) en la cuenca interna del Golfo Dulce (PROCMAR-Vida Silvestre Golfo Dulce-ISV Costa Rica). El enfoque analítico de esta investigación se desarrolló mediante dos aspectos claves:

a) Una comparación de la variable ecogeográfica profundidad máxima, la cual es relevante en la definición de los nichos tróficos en cetáceos (Forcada, 2002). Se contrastaron los valores de la profundidad máxima, asociados a la distribución espacial de los niveles tróficos (NT), tanto para las áreas marino-costeras de la Península de Osa, como para la cuenca interna del Golfo Dulce.

Se generó una matriz de datos sobre el nivel trófico y profundidad máxima, estratificada en tres subáreas: costera, plataforma y transición-oceánica, a partir de los mapas de distribución presentados por Oviedo et al. (in rev.) y Oviedo (2008). Los mapas rasterizados fueron contrastados con datos de profundidad máxima provenientes de una carta batimétrica digitalizada con una resolución de 1 a $12 \mathrm{~km}$, lo cual captura estructuras intermedias y de mayor tamaño de la cuenca oceánica. Las diferencias entre la profundidad máxima de cada nivel trófico en cada subárea y la zona proyectada para el establecimiento de la operación de maricultura en Punta Banco se evaluaron mediante la prueba Kruskal Wallis, asumiendo la distribución no paramétrica de los datos (Zar, 1996). El contraste se establece entre las áreas (Península de Osa-Cuenca Interna Golfo Dulce) de presencia significativa de depredadores NT 4.3 y 4.2 (Cuadro 1) y la zona designada para el establecimiento de las jaulas en términos de nichos tróficos reales, definidos por la profundidad máxima.

b) La proyección de los patrones de distribución mediante un modelo predictivo de base empírica, generado a partir de los registros de avistamiento de cetáceos en el Golfo Dulce. La información sobre la presencia de cetáceos se enfoca, en su mayoría, en dos especies: $S$. attenuata y T. truncatus. Estos registros se derivan de esfuerzos de investigación de carácter sistemático, cada avistamiento incluye información sobre fecha, hora, tamaño del grupo observado, conducta, condiciones 


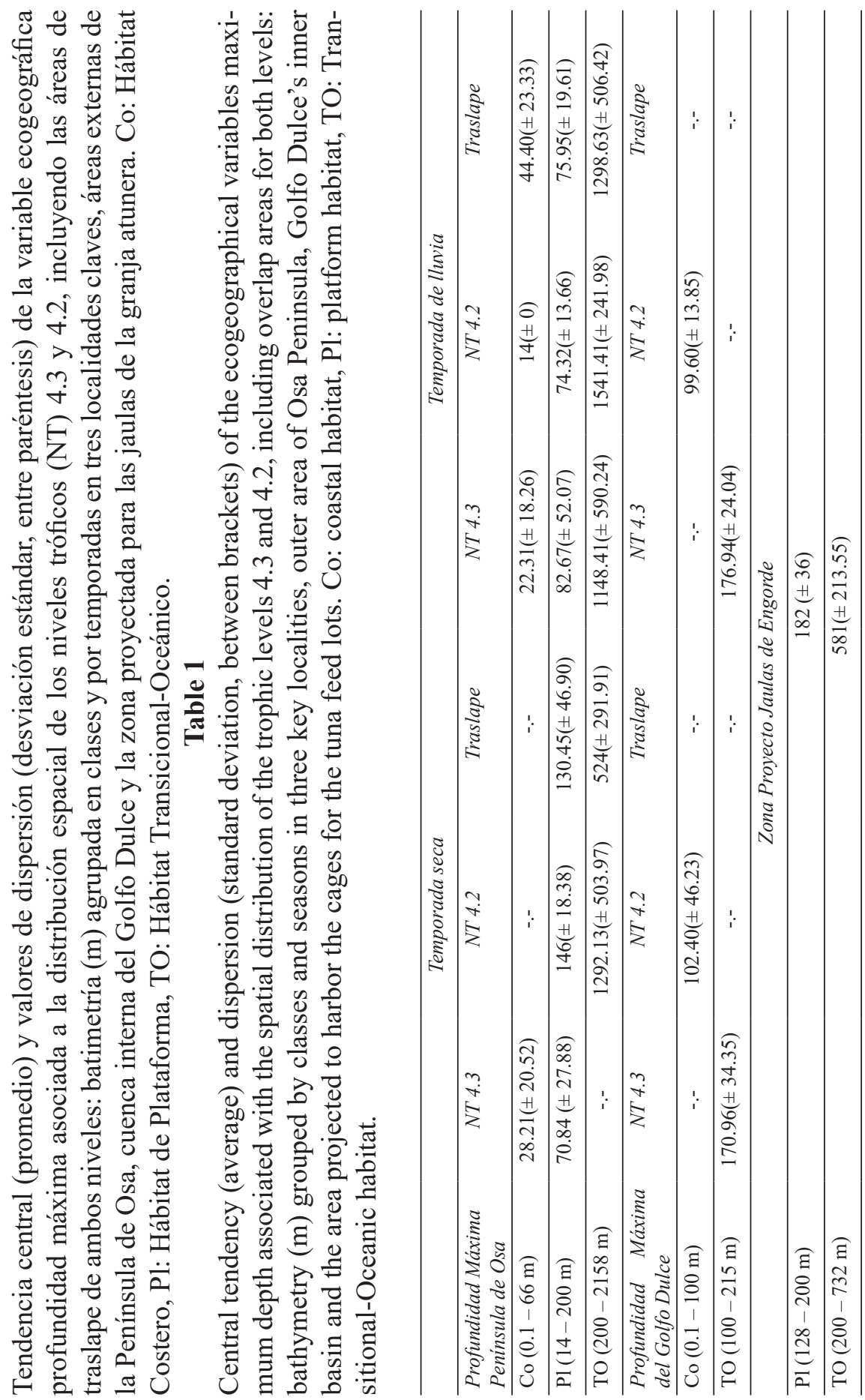


de detección basadas en la escala de fuerza en el viento Beaufort, posición geográfica (derivadas de lecturas de GPS) y estimados de abundancia relativa por medio de los índices de avistamiento y abundancia (Encuentros por Unidad de Esfuerzo: EPUE y Abundancia por Unidad de Esfuerzo: APUE, respectivamente), corregidos en función al esfuerzo en el campo expresado en el promedio mensual de tiempo invertido con avistamientos efectivos.

Los datos anteriores permitieron la elaboración de mapas con la distribución estacional de los niveles tróficos en el Golfo Dulce. Con detalles de la cuenca externa (datos generados por muestreos recientes), los mapas se produjeron utilizando una Interpolación Gaussiana Kringin, mediante la herramienta geoestadística del programa ArcGIS 9.2. Se incluyen adicionalmente avistamientos de ballenas jorobadas correspondientes a la subpoblación del Pacífico sudeste, con detalles de la posible ruta de ingreso a la cuenca externa del Golfo Dulce.

\section{RESULTADOS}

\section{Distribución de niveles tróficos y el área proyectada para las granjas atuneras}

Al considerarse y compararse la división de los ambientes marinos en la zona del Pacífico Sur en tres hábi- tats definidos con los valores de profundidad máxima, asociados al proyecto de jaulas de engorde, se establecen varias consideraciones claves:

1) No habría traslape entre los valores de profundidad máxima que definen el nicho trófico usado por depredadores de nivel 4.3 en aguas del hábitat de plataforma en las zonas marinas externas de la Península de Osa (en ambas temporadas: seca y de lluvia) y la zona proyectada para las jaulas de engorde. Esta diferencia en distribución de valores de profundidad máxima se sustenta estadísticamente (Kruskal Wallis, $\mathrm{X}^{2}: 15.90$ GL: $2, p<$ $0.05)$. Sin embargo, los valores de profundidad máxima asociados al mismo nivel trófico en la cuenca interna del Golfo Dulce, independientemente de la temporada, no presentan diferencias consistentes (Kruskal Wallis, $\mathrm{X}^{2}: 18.11$ GL: $2, p$ $>0.05$ ).

2)Las áreas de uso por parte de cetáceos del nivel trófico 4.2, durante la época seca dentro del hábitat de plataforma, se solaparían en los valores de profundidad máxima que definen el nicho ecológico, con las profundidades máximas asociadas a la zona del proyecto de jaulas de engorde en el mismo tipo de hábitat (Kruskal Wallis, $\mathrm{X}^{2}: 17.21$ GL: $2, p>0.05)$. Al considerarse los valores de profundidad máxima en la cuenca interna del Golfo Dulce, para el mismo nivel trófico, esta 
variable evidencia diferencias sustentadas estadísticamente (Kruskal Wallis, $\mathrm{X}^{2}: 15.24$ GL: $\left.2, p<0.05\right)$.

3)Las áreas incluidas en el hábitat transicional-oceánico dentro del complejo proyectado coinciden en los valores de profundidad máxima con los nichos ocupados durante la temporada seca, por cetáceos de ambos niveles tróficos (4.2-4.3), en el mismo hábitat en las zonas marino-costeras externas de la Península de Osa (Kruskal Wallis, $\mathrm{X}^{2}: 17.20$ GL: $2, p>0.05$ ). En la cuenca interna del Golfo Dulce no se observan traslapes entre ambos niveles tróficos.

\section{Distribución espacial de los regis- tros de cetáceos en el Golfo Dulce}

La distribución y densidad de cetáceos en el Golfo Dulce ilustra que en el nivel de la macroescala, el patrón de depredación es dominado por odontocetos de los niveles tróficos 4.3 y 4.2 , correspondientes a $S$. attenuata
(Figura 2a) y T. truncatus (Figura 2b), respectivamente (Cuadro 2).

En términos de diferencias estacionales, en la estación seca (Figura 3) se aprecia una clara distribución localizada de cada nivel trófico en bandas definidas, los delfines nariz de botella concentran su distribución en áreas de profundidad llana, dominando el ámbito costero de la cuenca interna y las aguas someras de la salida del Golfo, mientras los delfines manchados permanecen en aguas profundas de la cuenca interna y en zonas cercanas a la costa de la porción sur-oeste de la región externa, con progresión hacia aguas próximas a la isobata de los 200 $\mathrm{m}$.

Durante la temporada de lluvia (Figura 4), el patrón de localización discreto de los delfines manchados y nariz de botella se reemplazaría por un patrón de distribución mixto, particularmente en las áreas correspondientes a los hábitats costeros y de aguas profundas de la cuenca interna. Los delfines manchados se concentrarían más en la región central, con

\section{Cuadro 2}

Nombre común de todas las especies de odontocetos reportadas en este estudio, con detalles del nivel trófico asignado de acuerdo con Pauly et al. (1998).

\section{Table 2}

Common name of all odontocete species reported in this study, including details of assigned trophic level, after Pauly et al. (1998).

\begin{tabular}{ccc}
\hline Nombre común & Nombre científico & Nivel trófico (NT) \\
\hline Delfín manchado costero & Stenella attenuata graffmani & 4.3 \\
Delfín nariz de botella & Tursiops truncatus & 4.2 \\
Falsa orca & Pseudorca crassidens & 4.4 \\
\hline
\end{tabular}




\section{Figura 2}

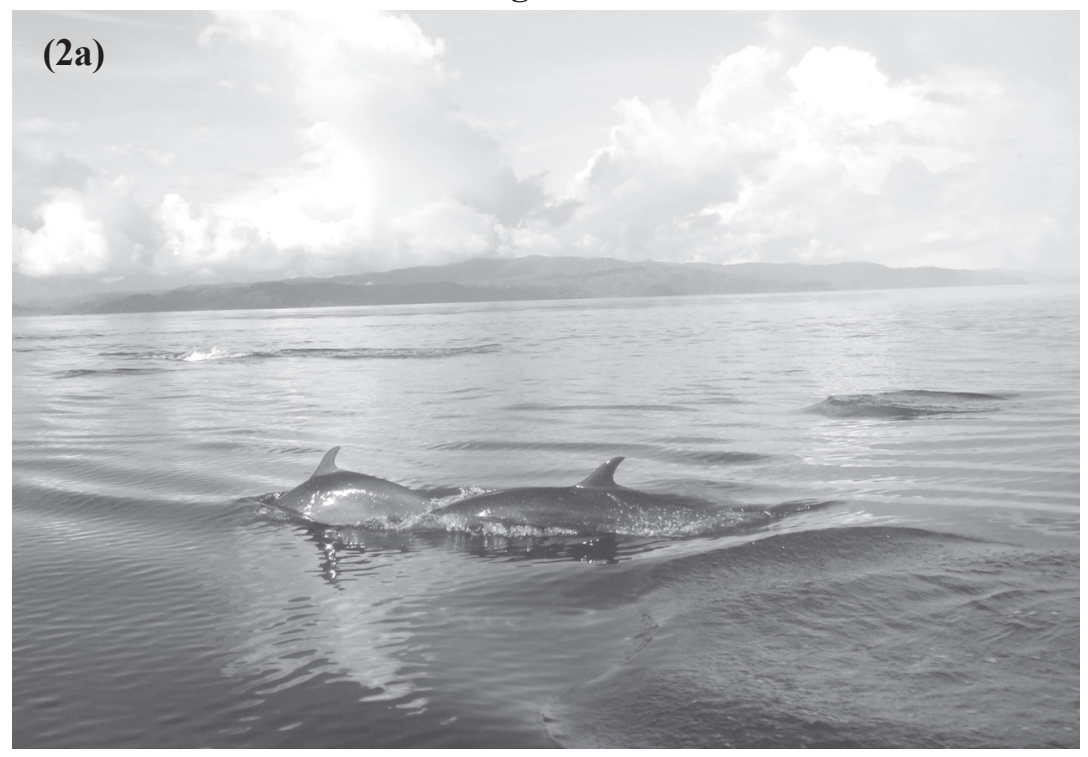

(2b)

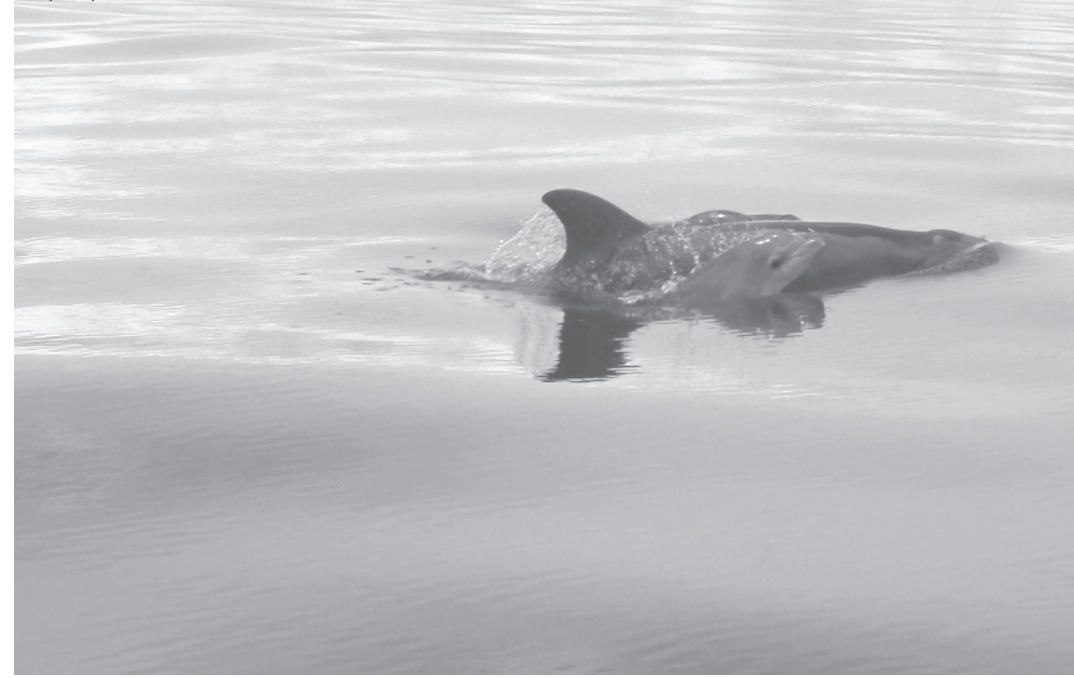

Avistamientos de cetáceos odontocetos y misticetos incluidos en los análisis de este estudio durante la temporada de lluvia (2009): delfín manchado (2a), delfín nariz de botella (2b).

Sightings of odontocete and mysticetes cetaceans during the rainy season (2009) sampling included in the analysis: pantropical spotted dolphin (2a), bottlenose dolphin (2b). 
Evaluación de los riesgos de afectación por el establecimiento de granjas atuneras en relación con la distribución espacial de cetáceos en el Golfo Dulce, Costa Rica

\section{Figura 2}

(2c)
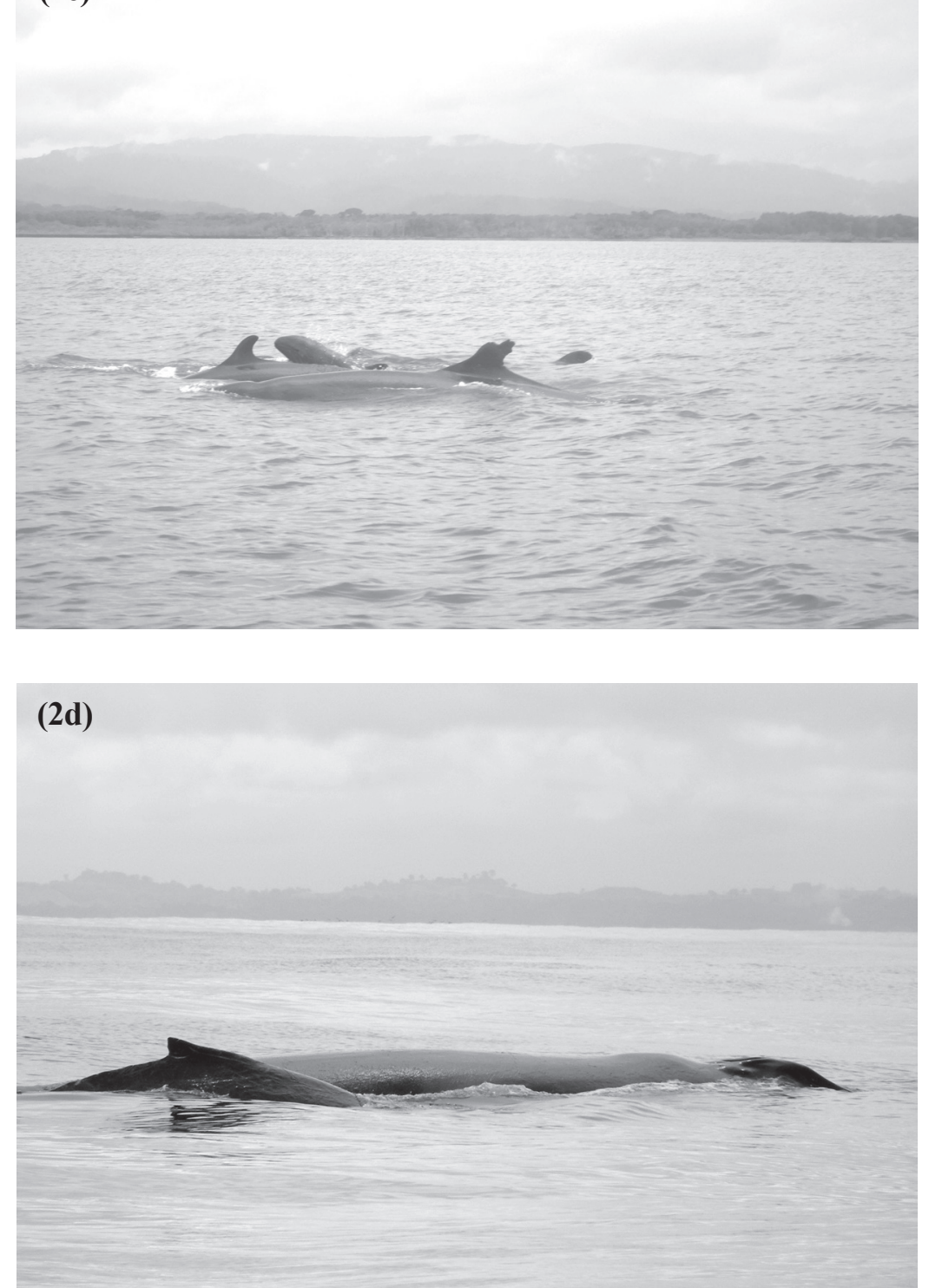

Avistamientos de cetáceos odontocetos y misticetos incluidos en los análisis de este estudio durante la temporada de lluvia 2009: falsa orca (2c) y ballena jorobada (2d).

Sightings of odontocete and mysticetes cetaceans during the rainy season 2009: false killer whale (2c), and humpback whale (2d). 
Figura 3

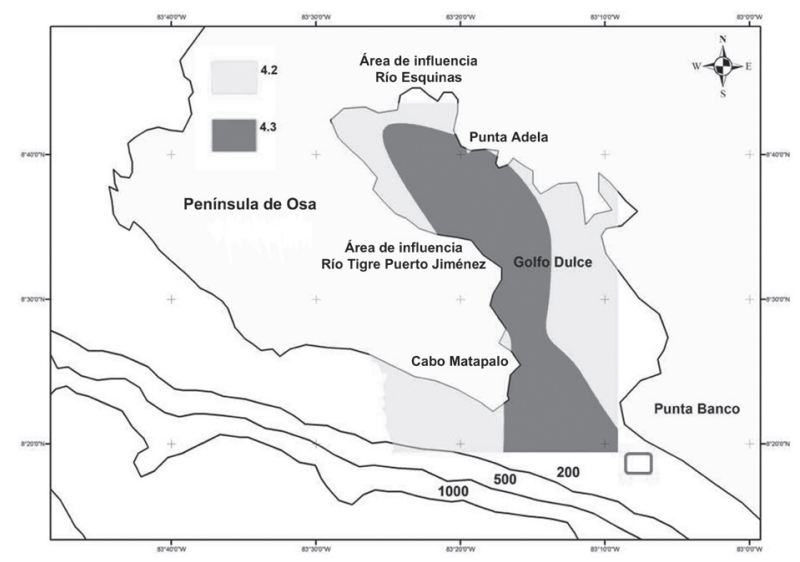

Distribución espacial de los niveles tróficos (NT: 4.2 y 4.3) de cetáceos odontocetos en el área de estudio durante la temporada seca (2009). La localización de las jaulas de engorde está representada por el rectángulo rojo.

Spatial distribution of odontocete cetacean trophic levels (NT: $4.2 \& 4.3$ ) in the study area during dry season (2009). Tuna feed lot facilities are represented by a red rectangle.

Figura 4

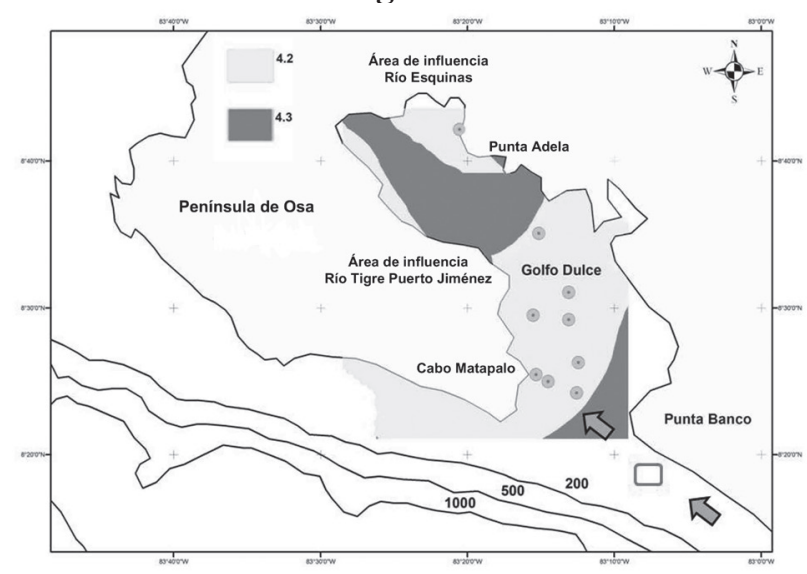

Distribución espacial de los niveles tróficos (NT: 4.2 y 4.3) de cetáceos odontocetos en el área de estudio durante la temporada de lluvia (2009). Adicionalmente se muestran avistamientos de ballenas jorobadas (círculos), incluyendo madres con crías y registros acústicos, así como la ruta migratoria potencial (desde áreas de alimentación en las costas de Chile y Antártica) de acceso al Golfo Dulce. La localización de las jaulas de engorde está representada por el rectángulo rojo.

Spatial distribution of odontocete cetaceans trophic levels (NT: $4.2 \& 4.3$ ) in the study area during dry season (2009). Additionally, humpback whales sightings, including mothers with calves and acoustic recordings are shown. Arrows are potential migration routes (from feeding grounds off Chile-Antarctica) to Golfo Dulce. Tuna feed lot facilities are represented by a red rectangle. 
presencia alterna en aguas cercanas a la costa, en localidades específicas como Punta Adela y en la zona de influencia de los cursos de aguas continentales mayores como El Río Tigre, cerca de Puerto Jiménez. Posteriormente habría una presencia notable de delfines manchados en las áreas aledañas a Punta Banco y que progresaría hacia aguas exteriores contiguas a la isobata de $\operatorname{los} 200 \mathrm{~m}$. Los delfines nariz de botella seguirían favoreciendo aguas más llanas y costeras, pero su presencia se incrementaría hacia la porción externa del Golfo Dulce, incluso bordeando la porción sur de la costa oeste de la Península de Osa. Adicionalmente, la presencia estacional de M. novaeangliae (Figura 2d) durante la temporada de lluvia se concentra sobre todo en la cuenca externa (Figura 4), con incursiones ocasionales hacia aguas más profundas en la zona interna del Golfo. La ruta migratoria de las ballenas jorobadas, con origen en Sudamérica, hace suponer que Punta Banco es una localidad de paso fijo, para acceder a aguas del Golfo Dulce.

\section{DISCUSIÓN}

\section{Distribución de los niveles tróficos de odontocetos}

El patrón de distribución de los niveles tróficos de cetáceos odontocetos descrito anteriormente ilustra el valor de los ecosistemas marinos y costeros del Pacífico Sur de Costa Rica, en la diversidad de depredadores topes. Esto tiene repercusiones importantes como medida de biodiversidad, así como de estabilidad y salud de los ecosistemas, considerando que la presencia de depredadores se relaciona directamente con la abundancia, así como con la riqueza de presas potenciales. Los flujos de energía e interacciones entre depredadores y presas son afectados por elementos de origen antrópico, que al parecer están todavía en niveles muy bajos en la zona en estudio.

La distribución de los niveles tróficos en el Golfo Dulce coincide con patrones espaciales previamente documentados para $S$. attenuata y $T$. truncatus (Acevedo-Gutiérrez \& Burkhart, 1998; Cubero-Pardo, 1998, 2007; Oviedo, 2007, 2008). También, el patrón de distribución concuerda con la repartición de hábitats de acuerdo con las características estructurales de los nichos ecológicos reales, que Oviedo (2007) estableció como la principal estrategia para la coexistencia de especies simpátricas. Esto previene áreas de traslape entre ambas especies, particularmente en la cuenca interna, lo que contrasta con la distribución de estos odontocetos en los ámbitos marinos y costeros de la región externa de la Península de Osa.

Es importante mencionar que depredadores de niveles tróficos altos (NT 4.4), como la falsa orca (Pseudorca crassidens, Figura 2c) ha sido reportada en aguas internas del Golfo Dulce (Acevedo-Gutiérrez, 1997), 
con encuentros recientes durante las temporadas de lluvia del 2008 y 2009. Las incursiones de $P$. crassidens en el Golfo Dulce podrían estar asociadas a la búsqueda de peces depredadores de gran talla. Durante los últimos encuentros se han documentado episodios de captura del pez gallo (Nematistius pectoralis).

\section{Distribución de niveles tróficos y el área proyectada para las granjas atuneras}

Los resultados presentados anteriormente reflejan cómo el riesgo de transposición de nichos por uso de hábitat potencial, es relevante $\mathrm{y}$ sustentado cuantitativamente. Esta tendencia que se sustenta en el traslape de la profundidad máxima, como variable ecogeográfica clave en la distribución de cetáceos y en la definición de nichos potencialmente explotables (Weir et al. 2001; Cañadas et al. 2002; Davis et al. 2002), evidencia una alta probabilidad de afectación a cetáceos odontocetos del nivel trófico 4.3 y 4.2, que corresponden particularmente al delfín manchado pantropical y al delfín nariz de botella. T. truncatus es la especie donde la interacción y afectación con complejos y operaciones de acuacultura ha sido ampliamente documentada (Díaz-López et al. 2005; Díaz-López, 2006 a, b; Díaz-López \& Bernal-Shirai, 2007).

Lo anterior implica que las jaulas serían un elemento que promovería el desplazamiento (no en términos de tránsito, sino de reemplazo) a corto plazo de las poblaciones de delfines que hacen uso del área, tal como está documentado en otras latitudes, en operaciones de cultivo similares con traslape extensivo de instalaciones de maricultura y especies de odontocetos residentes (Kemper et al. 2003; Heinrich, 2006; Ribeiro et al. 2007). Otro escenario de afectación se sostiene en el hecho, de que como todo depredador, estos delfines tenderían a buscar presas fáciles, lo que potenciaría el riesgo de captura incidental, es decir, delfines muertos en las redes de protección antidepredadores (Kemper \& Gibbs, 2001; Díaz-López \& Bernal-Shirai, 2007). Esta contingencia normal en las operaciones de maricultura fue responsable de la muerte de 29 individuos en el sur de Australia al interactuar con instalaciones de granjas de engorde de atún, en un período de 10 años, a un promedio de tres delfines por año (Kemper \& Gibbs, 2001). De acuerdo con Díaz-López \& Bernal-Shirai (2007), un patrón similar se ha documentado en Italia, en granjas de engorde de peces con alta incidencia de delfines nariz de botella en sus instalaciones. Las presas fáciles que atraerían a los delfines no serían precisamente la biomasa de peces en cultivo (en jaulas), sino la que se acumularía en las zonas externas adyacentes por el surplus de nutrientes en la columna de agua (Díaz-López et al. 2008). 
Las consecuencias anteriores son importantes en el impacto en las poblaciones locales de cetáceos, si se considera como ejemplo, a la población estimada de delfines nariz de botella en el Golfo Dulce, de aproximadamente 80 individuos según Acevedo \& Matthew (2005). Una reducción de 30 individuos en un período de 10 años, promovería un decrecimiento poblacional de $37.5 \%$ aproximadamente.

\section{El impacto de la granja atunera en las especies migratorias}

La presencia de especies migratorias y el riesgo de afectación por las granjas atuneras ha sido un argumento controversial, la parte interesada en la ejecución del proyecto contradijo en medios audiovisuales que habría impacto negativo en especies migratorias, particularmente en ballenas jorobadas, ya que la ruta migratoria no incluía Punta Banco. Dicha afirmación se basó en censos hechos en su mayoría durante la temporada correspondiente al invierno boreal, donde el origen de la migración es en latitudes boreales. La afirmación anterior pasa por alto la presencia de ballenas jorobadas de la población del Pacífico sur-oriental, ambas subpoblaciones, la del hemisferio norte y sur, utilizan las aguas costeras de Costa Rica como zonas de reproducción y cría (Acevedo-Gutiérrez \& Smultea, 1995; May-Collado et al. 2005), particular- mente las áreas marino-costeras de la Península de Osa (Oviedo \& Solís, 2008), incluyendo las aguas internas del Golfo Dulce (Acevedo-Gutiérrez \& Smultea, 1995), tal como lo confirman los registros recientes detallados anteriormente (incluyendo registros acústicos de machos cantando). Naturalmente, si el punto de origen en la migración de las ballenas jorobadas del Pacífico sudeste incluye la costa oeste de Suramérica, el paso y acceso de esta subpoblación para ingresar al Golfo Dulce, incluiría las aguas aledañas a Punta Banco.

El establecimiento de infraestructura mayor, como las jaulas de maricultura, podría en efecto constituir un elemento de perturbación, alteración e incluso modificación de conductas relacionadas con los movimientos migratorios.

En conclusión, el área propuesta para el establecimiento de las jaulas de engorde se ubicaría en una zona potencial de presencia significativa de cetáceos, incluyendo depredadores de niveles tróficos superiores como la falsa orca y especies migratorias como las ballenas jorobadas, con un riesgo notable de transposición entre las jaulas y el hábitat potencial de cetáceos en términos de profundidad y particularmente del nicho de alimentación. Lo anterior también implica una alta probabilidad de captura incidental de delfines por la interacción con las instalaciones, ya que las jaulas inducirían al incremento de fauna acompañante 
detritívora, que serían presas de fácil accesibilidad para los delfines y otros depredadores.

La recomendación más importante que se genera de las conclusiones anteriores sería considerar el principio precautorio en conjunto con lo establecido por la legislación en términos de conservación de mamíferos marinos y no permitir el establecimiento de la operación en una zona de tanta importancia en términos de biodiversidad marina, en particular de alta riqueza en depredadores marinos topes. La reubicación del proyecto en otras zonas costeras menos prístinas podría ser una alternativa.

\section{AGRADECIMIENTOS}

Los datos utilizados en esta contribución fueron recolectados gracias al apoyo de la Fundación Vida Marina, Fundación Evergreen en Península de Osa e Isla del Caño y PROCMAR, International Students Volunteers Costa Rica (ISV Costa Rica) en el Golfo Dulce. Las fotos de la figura 2 fueron tomadas por Lilianne Steele (2a), Sarah Monks (2b) y Chantal Lyons (2c).

\section{BIBLIOGRAFÍA}

Acevedo-Gutiérrez, A. (1997). Resightings and behavior of false killer whales (Pseudorca crassidens) in Costa Rica. Mar. Mam. Sci., 13, 307-314.

Acevedo-Gutiérrez, A. \& Smultea, M. A. (1995). First records of humpback whales including calves at Golfo Dulce and Isla del Coco, Costa Rica, suggesting geographical overlap of northern and southern Hemisphere populations. Mar. Mam. Sci., 11, 554-560.

Acevedo-Gutiérrez, A. \& Burkhart, S. (1998). Seasonal distribution of bottlenose (Tursiops truncatus) and pan-tropical spotted (Stenella attenuata) dolphins (Cetacea: Delphinidae) in Golfo Dulce, Costa Rica. Rev. Biol. Trop., 46, 91-101.

Acevedo-Gutiérrez, A. \& Matthew, A. (2005). Association pattern of bottlenose dolphins in Costa Rica: Constant Companions and Casual Acquaintances. Book of abstract XVI Biennial Conference on the Biology of Marine Mammals, San Diego, California.

Begon, M., Townsend, C. R. \& Harper, J. L. (2006). Ecology: from individuals to ecosystems. 4th ed. Blackwell Publishing, U.K. 740 pp. Cañadas, A., Sagarminaga, R. \& García-Tiscar, S. (2002). Cetacean distribution related with depth and slope in Mediterranean waters off sourthern Spain. Deep Sea Res., I, 49, 2053-2073.

Cubero-Pardo, P. (1998). Patrones de comportamiento diurnos y estacionales de Tursiops truncatus y Stenella attenuata (Mammalia: Delphinidae) en el Golfo Dulce, Costa Rica. Rev. Biol. Trop., 46, 103-110. 
Cubero-Pardo, P. (2007). Distribución y condiciones ambientales asociadas al comportamiento del delfín bufeo (Tursiops truncatus) y el delfín manchado (Stenella attenuata) (Cetacea: Delphinidae) en el Golfo Dulce, Costa Rica. Rev. Biol. Trop., 55, 549-557.

Davis, R. W., Ortega Ortiz, J. G., Ribie, C. A., Evans, W. E., Biggs, C. D., Ressler, P. H., Cady, R. B., Leben, R. R., Mullin, D. K \& Würsig, B. (2002). Cetacean habitat in the northern oceanic Gulf of Mexico. Deep Sea Res., I, 49, 121-142.

Díaz-López, B., Marini, L. \& Polo, F. (2005). The impact of a fish farm on a bottlenose dolphin population in the Mediterranean Sea. Thalassas, 21, 53-58.

Díaz-López, B. (2006a). Interactions between Mediterranean bottlenose dolphins (Tursiops truncatus) and gillnets off Sardinia, Italy. ICES J. Mar. Sci., 63 (5), 775-960.

Díaz-López, B. (2006b). Bottlenose dolphin (Tursiops truncatus) predation on a marine finfish farm: some underwater observations. Aquat. Mammals, 32, 305-310.

Díaz-López, B. \& Bernal-Shirai, J. A. (2007). Bottlenose dolphin (Tursiops truncatus) presence and incidental capture in a marine fish farm on the north-eastern coast of Sardinia (Italy). J. Mar. Biol. Assoc. UK, 87, 113-117.

Díaz-López, B., Bunke, M. \& Bernal-Shirai, J. A. (2008). Mari- ne aquaculture off Sardinia Island (Italy): ecosystem effects evaluated through a trophic mass-balance model. Ecol. Model., 212, 292-303.

Evans, P. G. H. (2002). Habitat pressures, pp. 545-548. In: Perrin, W. F., Wursig, B. \& Thewissen, J. G. M. (eds.). Encyclopedia of Marine Mammals. San Diego: Academic Press.

Fernández, M., Oviedo, L., Echeverria, D. \& Goodman, S. (2007). Co-occurrence of cetaceans indicator species of up-welling modified waters and tropical warm waters off Osa Peninsula, Costa Rica. Paper presented at the Seventeenth Biennial Conference on the Biology of Marine Mammals, Cape Town, South Africa (Available from www.vidamarina.org). Fernández, M. \& Oviedo, L. (2009). Distribution and abundance of Delphinus delphis off the southern Pacific Coast of Costa Rica. Paper SC/SM3 presented to the IWC 60th Scientific Committee, June, Madeira, Portugal.

Fieldler, P. (2002). Ocean environment, pp. 824-830. In: Perrin, W. F., Wursig, B. \& Thewissen, J. G. M. (eds.). Encyclopedia of Marine Mammals. San Diego: Academic Press.

Forcada, J. (2002). Distribution, pp. 327-333. In: Perrin, W. F., Wursig, B. and Thewissen, J. G. M. (eds.). Encyclopedia of Marine Mammals. San Diego: Academic Press. 
Heinrich, S. (2006). Ecology of Chilean dolphins and Peale's dolphins at Isla Chiloé, southern Chile. PhD Thesis. University of St Andrews. Scotland.

Kemper, C. M. \& Gibbs, S. E. (2001). Dolphin interactions with tuna feedlots at Port Lincoln, South Australia and recommendations for minimizing entanglements. $J$. Cetacean Res. Manage., 3, 283292.

Kemper, C. M., Pemberton, D., Cawthorn, M., Heinrich, S., Mann, J., Würsig, B., Shaughnessy, P. \& Gales, R. (2003). Aquaculture and marine mammals: co-existence of conflict? In: Marine mammals: $f$ sheries, tourism and management issues (ed. N. Gales et al.), pp. 208-225. Collingwood, Victoria, Australia: CSIRO Publishing.

May-Collado, L., Gerrodette, T., Calambokidis, J., Rasmussen, K. \& Sereg, I. (2005). Patterns of cetacean sighting distribution in the Pacific Exclusive Economic Zone of Costa Rica, based on data collected from 1979-2001. Rev. Biol. Trop., 53, 249-263.

Oviedo, L. (2007). Dolphin sympatric ecology in a tropical fjord: habitat partitioning by bathymetry and topography as a strategy to coexist. J. Mar. Biol. Asso. UK, $87,1-9$.

Oviedo, L. (2008). Análisis del uso de hábitat del delfín manchado pantropical Stenella attenuata (Cetacea: Delphinidae) en el
Golfo Dulce, Costa Rica. MSc. Thesis. Universidad Nacional (UNA), Puntarenas, Costa Rica.

Oviedo, L. \& Solís, M. (2008). Underwater topography determines critical breeding habitat for humpback whales near Osa Peninsula, Costa Rica: implications for Marine Protected Areas. Rev. Biol. Trop., 52, 591-602.

Oviedo, L., Fernández, M. \& Pacheco, J. D. (in rev.). Trophic levels distribution of toothed cetaceans in the Southern Pacific Coast of Costa Rica.

Pauly, D., Trites, A. W., Capuli, E. \& Christensen, V. (1998). Diet composition and trophic levels of marine mammals. ICES J. Mar. Sci., 55, 467-481.

Ramesh, B. R. (2008). Forest landscape approach for biodiversity management in the Western Ghats. J. Lands, Archit., 5, 33-37. Ribeiro, S., Viddi, F. A., Cordeiro, J. L. \& Freitas, T. R. O. (2007). Fine scale habitat selection of Chilean dolphins (Cephalorhynchus eutropia): interactions with aquaculture activities in southern Chiloé Island, Chile. J. Mar. Biol. Asso. UK, 87, 119-128.

Weir, C. R., Pollock, C., Cronin, C. \& Taylor, S. (2001). Cetaceans of the Atlantic Frontier, north and west of Scotland. Cont. Shelf Res., 21, 1047-1071.

Zar, J. H. (1996). Biostatistical analysis. London: Prentice-Hall International (UK) Ltd. 420 pp. 